\title{
Recanalized Aneurysms: Is Rupture Further Preventable?
}

l: read with great interest the article by Funakoshi et al, "Predictors of Cerebral Aneurysm Rupture after Coil Embolization: SingleCenter Experience with Recanalized Aneurysms." They reviewed their results of coil embolization of unruptured and ruptured saccular cerebral aneurysms retrospectively and analyzed the rate of recanalization and bleeding from treated aneurysms.

It is noteworthy that $44.1 \%$ of treated unruptured aneurysms are paraclinoid aneurysms, which usually account for less than $1 \%$ of SAH in Japan. ${ }^{2}$ Presumably, their institution has a lot of referrals for difficult paraclinoid cases, but considering the realworld frequency of the origin of SAH cases, the risk of bleeding from paraclinoid aneurysms should be small. Hence, while I reserve a small leap in argument, it makes sense to assume that filling this low-risk aneurysm with coils will not result in enlargement or bleeding, though some paraclinoid aneurysms recanalized in their Results section.

The authors calculated the rupture risk after coil embolization to be $0.20 \% / y e a r$ by a person-year method. If the number of lowrisk aneurysms, ie, paraclinoid aneurysms, increases in the cohort, the observation period without recanalization or rupture becomes large and results in smaller apparent bleeding and recanalization rates. Therefore, aneurysms that cause SAH in actual clinical practice and these low-risk aneurysms, like paraclinoid aneurysms, should be better analyzed separately, though they are usually discussed in a mixed bag.

The authors claim that coiled aneurysms with class IIIb recanalization should have an early retreatment from analysis of rupture after coil embolization, which would be reasonable. As is mentioned in the Discussion section, those aneurysms that showed new blebs were treated, and more than $40 \%$ of all recanalized aneurysms were retreated. The ruptures occurred in the remaining

http://dx.doi.org/10.3174/ajnr.A6734 patients. Five of 8 ruptures after coil embolization seem to have occurred in patients with $\mathrm{mRS}>3$ (Table 2), which might have affected the indication of retreatment, and the rupture may be a simple result of "natural history" of observation of recanalized aneurysms.

As they mentioned in the Limitations section, cases of the ruptured group with severe neurologic deficit were not followed up, and the information is limited, which is not to be blamed. However, they should have referred to the grade of SAH and provided the survival curve with the number at risk because the mean follow-up duration of the ruptured group is much shorter than that of the unruptured group, which may be the result of the percentage of patients with poor grades. If the number of patients followed up differs substantially, the interpretation of the results needs to be kept in mind. In addition, the maximum duration of recanalized aneurysms is longer than that of all aneurysms in Table 1. I wonder if this was interquartile range or range.

Last, I appreciate the authors for frankly presenting their clinical results. It will contribute to the discussion about treatment decisions for cerebral aneurysms.

\section{REFERENCES}

1. Funakoshi $Y$, Imamura H, Tani S, et al. Predictors of cerebral aneurysm rupture after coil embolization: single-center experience with recanalized aneurysms. AJNR Am J Neuroradiol 2020;41:828-35 CrossRef Medline

2. Yoshiteru Tada SN. Classification of subarachnoid hemorrhage severity and frequency by aneurysm location and CT findings.. In: Kobayashi S, ed. Japanese Stroke Data Bank 2015. Nakayama Shoten Co, Ltd; 2015:152-53
(D)T. Kimura

Department of Neurosurgery Japanese Red Cross Medical Center Tokyo, Japan 